\title{
Combinatorial Gelfand models for some semigroups and $q$-rook monoid algebras
}

\author{
Ganna Kudryavtseva and Volodymyr Mazorchuk
}

\begin{abstract}
Inspired by the results of APR, we propose combinatorial Gelfand models for semigroup algebras of some finite semigroups, which include the symmetric inverse semigroup, the dual symmetric inverse semigroup, the maximal factorizable subsemigroup in the dual symmetric inverse semigroup, and the factor power of the symmetric group. Furthermore we extend the Gelfand model for the semigroup algebras of the symmetric inverse semigroup to a Gelfand model for the $q$-rook monoid algebra.
\end{abstract}

\section{Introduction}

Let $A$ be a finite-dimensional unital associative algebra over $\mathbb{C}$ and $M$ be an $A$-module. The module $M$ is said to be a Gelfand model for $A$ if it is isomorphic to a multiplicity-free direct sum of all simple $A$-modules. The present paper is inspired by the results of [APR], where beautiful combinatorial Gelfand models for the group algebra $\mathbb{C} S_{n}$ of the symmetric group, and for the corresponding Hecke algebra $\mathbf{H}_{n}(q)$ are constructed. We refer the reader to the list of references in [APR] for the history of the question and an account on known Gelfand models.

The aim of the present paper is to extend the results of APR to some classes of finite semigroups, which include several inverse generalizations of the symmetric group, in particular, the full symmetric inverse semigroup $\mathcal{I S}_{n}$; and to the Hecke algebra analogue for $\mathcal{I S}_{n}$, known as the $q$-rook monoid algebra. The latter one has been recently defined by Solomon, $\mathrm{So}$, however, a special case already appeared in So1. The $q$-rook monoid algebra has been studied by several authors, see [DHP, $[\mathrm{HR},[\mathrm{Ha},[\mathrm{Pa},[\mathrm{So}]$ and references therein. Our motivation comes from an attempt to better understand the connection between the combinatorial and representation theoretical properties of these objects. 
The paper is organized as follows: in Section 2 we recall the combinatorial Gelfand model for $\mathbb{C} S_{n}$, constructed in APR. In Section 3 we show how the latter model can be used to construct combinatorial Gelfand models for semigroup algebras of those finite semigroups, for which each trace of a regular $\mathcal{D}$-class is an inverse semigroup in which maximal subgroups are direct sums of symmetric groups. Examples of such semigroups include the symmetric inverse semigroup (see [GM3, 2.5]), the dual symmetric inverse semigroup (see $[\mathrm{FL}]$ ), and the maximal factorizable subsemigroup in the dual symmetric inverse semigroup (see [FL]). Another, rather surprising, natural example is the factor power of the symmetric group (see [GM1]), which, in particular, is not even regular. In Section 4 we recall (an appropriate modification of) the combinatorial Gelfand model for the Hecke algebra $\mathbf{H}_{n}(q)$, constructed in [APR. Finally, in Section 5 we extend the latter model to a combinatorial Gelfand model for the $q$-rook monoid algebra $\mathbf{I}_{n}(q)$ from [So]. For $\mathbf{I}_{n}(q)$ we use the presentation from [Ha], which is different from the one used in $\mathrm{SO}$.

Acknowledgment. This work was done during the visit of the first author to Uppsala University, which was supported by the Royal Swedish Academy of Sciences (KVA) and the Swedish Foundation for International Cooperation in Research and Higher Education (STINT). For the second author the research was partially supported by the Swedish Research Council. The financial support of the Swedish Research Council, KVA and STINT and the hospitality of Uppsala University are gratefully acknowledged. We thank Rowena Paget for her remarks on the original version of the paper, especially on Section 2. We also thank the referee for very useful remarks.

\section{Combinatorial Gelfand model for $\mathbb{C} S_{n}$}

Let $S_{n}$ be the symmetric group on $\{1,2, \ldots, n\}$ and $\mathcal{I}_{n}$ be the set of all involutions in $S_{n}$ (recall that $\pi \in S_{n}$ is an involution provided that $\pi^{2}=\mathrm{id}$, in particular, the identity element id itself is an involution). For $\pi \in S_{n}$ we define the inversion set of $\pi$ as follows:

$$
\operatorname{Inv}(\pi)=\{(i, j): i<j \text { and } \pi(i)>\pi(j)\} .
$$

For $w \in \mathcal{I}_{n}$ set

$$
\operatorname{Pair}(w)=\{(i, j): i<j \text { and } w(i)=j\} .
$$

Set $\operatorname{Inv}_{w}(\pi)=\operatorname{Inv}(\pi) \cap \operatorname{Pair}(w)$ and $\operatorname{inv}_{w}(\pi)=\left|\operatorname{Inv}_{w}(\pi)\right|$. Finally, let $V_{n}$ be the vector space with the basis $\left\{\mathrm{I}_{w}: w \in \mathcal{I}_{n}\right\}$. 
Theorem 1 ([APR $])$. The assignment

$$
\pi \cdot \mathrm{I}_{w}=(-1)^{\operatorname{inv}_{w}(\pi)} \mathrm{I}_{\pi w \pi^{-1}}, \quad \pi \in S_{n}, w \in \mathcal{I}_{n}
$$

defines on $V_{n}$ the structure of a $\mathbb{C} S_{n}$-module. Moreover, this module is a Gelfand model for $\mathbb{C} S_{n}$.

Remark 2. Theorem 1 extends to direct sums of symmetric groups in a straightforward way.

Remark 3. For $k=0,1, \ldots,\left\lfloor\frac{n}{2}\right\rfloor$ let $\mathcal{I}_{n}^{k}$ denote the subset of $\mathcal{I}_{n}$ consisting of all involutions, which can be written as a product of exactly $k$ pairwise different and commuting transpositions. We obviously have that $\mathcal{I}_{n}$ is a disjoint union of the $\mathcal{I}_{n}^{k}$-s. Moreover, the linear span $V_{n}^{k}$ of $\left\{\mathcal{I}_{w}: w \in \mathcal{I}_{n}^{k}\right\}$ is invariant under the $\mathbb{C} S_{n}$-action for every $k$. The Robinson-Schensted correspondence, [Sa, 3.1], assigns to each $\pi \in S_{n}$ a pair $(a(\pi), b(\pi))$ of standard Young tableaux of the same shape. Moreover, $\pi \in S_{n}$ is an involution if and only if $a(\pi)=b(\pi)$, [Sa, Theorem 3.6.6]. Using the properties of Viennot's shadow diagrams (see $[\mathrm{Sa}, 3.6]$ ) one shows that two elements $w, w^{\prime} \in \mathcal{I}_{n}$ belong to the same $\mathcal{I}_{n}^{k}$ provided that $a(w)$ and $a\left(w^{\prime}\right)$ have the same shape. Using the main result of [RS and tensoring with the sign representation one further shows that $V_{n}^{k}$ is isomorphic to the direct sum of Specht modules $S^{\lambda}$, where $\lambda$ runs through the set of all shapes of $a(w)$ for $w \in \mathcal{I}_{n}^{k}$.

\section{Combinatorial Gelfand models for semi- group algebras of some finite semigroups}

We use [Hi] or GM3] as general reference for standard notions from semigroup theory. Let $S$ be a finite semigroup and $E(S)$ its set of idempotents. For $e \in E(S)$ consider the $\mathcal{D}$-class $\mathrm{D}_{e}$ containing $e$. Then $\mathrm{D}_{e} \cup\{0\}$ with multiplication given by

$$
a \star b= \begin{cases}a b, & a b \in \mathrm{D}_{e} \\ 0, & \text { otherwise }\end{cases}
$$

is called the trace of $\mathrm{D}_{e}$. From now on we assume that for every $e \in E(S)$

- the trace $\mathrm{D}_{e} \cup\{0\}$ is an inverse semigroup,

- the maximal subgroup $G_{e}$ of $S$, corresponding to $e$, is a direct sum of symmetric groups. 
Let $e_{1}, \ldots, e_{k} \in E(S)$ be a fixed collection of idempotents, one for each $\mathcal{D}$ class. Let further $m_{i}, i=1, \ldots, k$, denote the number of $\mathcal{L}$-classes inside $\mathrm{D}_{e_{i}}$. For each $i=1, \ldots, k$ we fix an isomorphism of the group $G_{e_{i}}$ with $S_{n_{1}^{(i)}} \oplus \cdots \oplus S_{n_{l_{i}}^{(i)}}$ and an isomorphism $\varphi_{i}$ of $\mathrm{D}_{e_{i}} \cup\{0\}$ with the Brandt semigroup associated with the group $S_{n_{1}^{(i)}} \oplus \cdots \oplus S_{n_{l_{i}}^{(i)}}$ and the cardinality $m_{i}$ (for details see [CP, §3.3]). This means that we have $\varphi_{i}(0)=0$ and for any $x \in \mathrm{D}_{e_{i}}$ we have $\varphi_{i}(x)=(a, y, b)$, where $y \in S_{n_{1}^{(i)}} \oplus \cdots \oplus S_{n_{l_{i}}^{(i)}}$, and $a, b \in\left\{1, \ldots, m_{i}\right\}$. The multiplication in the Brandt semigroup is given by

$$
(a, y, b) \star\left(a^{\prime}, y^{\prime}, b^{\prime}\right)= \begin{cases}\left(a, y y^{\prime}, b^{\prime}\right), & b=a^{\prime} \\ 0, & \text { otherwise }\end{cases}
$$

We set $\bar{\varphi}_{i}(x)=y$.

An element $w \in S$ will be called an involution provided that $w \in G_{e}$ for some $e \in E(S)$ and $w^{2}=e$. Let $\mathcal{I}_{S}$ denote the set of all involutions in $S$, and $V_{S}$ the vector space with the basis $\left\{\mathrm{I}_{w}: w \in \mathcal{I}_{S}\right\}$. Our first result is the following:

Theorem 4. Let $x \in S$ and $w \in \mathcal{I}_{S}$ be such that $w \in G_{e}$ for some $e \in E(S)$ and $e \in \mathrm{D}_{e_{i}}$ for some $i \in\{1,2, \ldots, k\}$. Then the assignment

$$
x \cdot \mathrm{I}_{w}= \begin{cases}(-1)^{\operatorname{inv}_{\bar{\varphi}_{i}(w)}\left(\bar{\varphi}_{i}(x e)\right)} \mathrm{I}_{(x e) w(x e)^{-1},} & \text { xe } \in \mathrm{D}_{e} ; \\ 0, & \text { otherwise }\end{cases}
$$

defines on $V_{S}$ the structure of a $\mathbb{C} S$-module. Moreover, this module is a Gelfand model for $\mathbb{C} S$.

Proof. Let $x, y \in S$ be such that $x \cdot\left(y \cdot \mathrm{I}_{w}\right)=0$. We show that in this case we also have $x y \cdot I_{w}=0$. Suppose first that $y \cdot I_{w}=0$. Let $\leq_{\mathcal{J}}$ denote the natural partial order on $S$ associated with Green's $\mathcal{J}$-relation. Then $y e<_{\mathcal{J}} e$ and thus $x y e \leq_{\mathcal{J}} y e<_{\mathcal{J}} e$, which means that $x y \cdot \mathrm{I}_{w}=0$. Suppose now that $y \cdot \mathrm{I}_{w} \neq 0$, but $x \cdot\left(y \cdot \mathrm{I}_{w}\right)=0$. Denote $v=(y e) w(y e)^{-1}$. Let $f$ be an idempotent such that $v \in G_{f}$. Then $x \cdot \mathrm{I}_{v}=0$, which implies that $x f<_{\mathcal{J}} f$. The definition of $f$ yields that $f y e \mathcal{R} y e$, which, in turn, gives us that $f y e=y e$. Thus we have

$$
x y e=x f y e \leq_{\mathcal{J}} x f<_{\mathcal{J}} f .
$$

It follows that $x y \cdot \mathrm{I}_{w}=0$ as well.

Let now $x, y \in S$ be such that $x \cdot\left(y \cdot \mathrm{I}_{w}\right) \neq 0$. Denote $G=G_{e_{i}}$ and set $\bar{x}=\bar{\varphi}_{i}(x)$ for every $x \in \mathrm{D}_{e_{i}}$. Without loss of generality we will assume 
that $\varphi_{i}\left(G_{e}\right)=(1, G, 1)$ and that $\bar{g}=g$ whenever $g \in G_{e}$. Since $y \cdot \mathrm{I}_{w} \neq 0$ it follows that ye $\mathcal{J} e$ and thus ye $\mathcal{L} e$. In particular, $\varphi_{i}(y e)=(l, \overline{y e}, 1)$ for some $l$. Then we have $\varphi_{i}\left((y e)^{-1}\right)=\left(1, \overline{y e}^{-1}, l\right)$, where $\overline{y e}^{-1}$ is the inverse of $\overline{y e}$ in $G$. Let $f=(y e)(y e)^{-1}$ and $v=(y e) w(y e)^{-1}$. Then $\varphi_{i}(f)=\left(l, e_{i}, l\right)$ and $\varphi_{i}(v)=\left(l, \overline{y e} w \overline{y e}^{-1}, l\right)$. Since $x \cdot \mathrm{I}_{v} \neq 0$ it follows that $x f \mathcal{J} f$ and thus $x f \mathcal{L} f$. Set $u=(x f)(y e) w(y e)^{-1}(x f)^{-1}$. Applying the equality $x f y e=x y e$ one shows that $\varphi_{i}(u)=\left(k, \overline{x y e} w \overline{x y e}^{-1}, k\right)$ for some $k$.

Now the first part of the proof amounts to checking the equality

$$
(-1)^{\operatorname{inv}_{w}(\overline{y e})} \cdot(-1)^{\operatorname{inv}_{\overline{y e} w \overline{y e}}-1(\overline{x f})}=(-1)^{\operatorname{inv}_{w}(\overline{x y e})} .
$$

Let $(i, j) \in \operatorname{Pair}(w)$. Suppose that $(i, j) \in \operatorname{Inv}_{w}(\overline{y e})$, that is $i<j$ and $\overline{y e}(i)>\overline{y e}(j)$. If $\overline{x f}(\overline{y e}(i))<\overline{x f}(\overline{y e}(j))$ then we have that $(\overline{y e}(j), \overline{y e}(i))$ belongs to $\operatorname{Inv}_{\overline{y e} w \overline{y e}}(\overline{x f})$, and at the same time $(i, j) \notin \operatorname{Inv}_{w}(\overline{x y e})$. If $\overline{x f}(\overline{y e}(i))>\overline{x f}(\overline{y e}(j))$ then $(\overline{y e}(j), \overline{y e}(i))$ does not belong to $\left.\operatorname{Inv}_{\overline{y e} w \overline{y e}}-1 \overline{x f}\right)$, and at the same time $(i, j) \in \operatorname{Inv}_{w}(\overline{x y e})$. Analogously one considers the case $(i, j) \notin \operatorname{Inv}_{w}(\overline{y e})$, and (2) follows. Therefore $V_{S}$ is indeed a $\mathbb{C} S$-module.

We are left to show that $V_{S}$ is a Gelfand model for $S$. We will use the fact that simple modules over the complex semigroup algebra of a finite semigroup $S$ are in bijective correspondence with simple modules of $G_{e_{i}}, 1 \leq i \leq k$ (see [CP, Chapter 5] or [GMS, Theorem 7] for a more modern approach). In view of this, it is enough to show that for a maximal subgroup $G$ of $S$ and a simple $\mathbb{C} G$-module $V$ the corresponding $\mathbb{C} S$-module $V^{\prime}$ is isomorphic to a submodule of $V_{S}$, and then to make sure that the sum of the dimensions of all $V^{\prime}$-s equals the dimension of $V_{S}$.

Let $1 \leq i \leq k$ and $\mathcal{I}_{n, i}$ be the set of involutions contained in maximal subgroups of the $\mathcal{D}$-class $\mathrm{D}_{e_{i}}$. Then the linear span $V_{S}^{i}$ of all $\mathrm{I}_{w}, w \in \mathcal{I}_{n, i}$, is a direct summand of $V_{S}$. The dimension of this direct summand equals $m_{i} \cdot\left|\left\{I_{w}: w \in \mathcal{I}_{G_{e_{i}}}\right\}\right|$. The action of $G_{e_{i}}$ on the linear span $V\left(G_{e_{i}}\right)$ of $\left\{\mathrm{I}_{w}: w \in \mathcal{I}_{G_{e_{i}}}\right\}$, coinsides with the action from [APR], and thus from [APR, Theorem 1.1.2] and Remark 2 it follows that $V\left(G_{e_{i}}\right)$ is a multiplicityfree direct sum of all simple $G_{e_{i}}$-modules. Let $V$ be a simple direct summand of $V\left(G_{e_{i}}\right)$ (as a $G_{e_{i}}$-module). Suppose that the image of $G_{e_{i}}$ under $\varphi_{i}$ is $\left(1, G_{e_{i}}, 1\right)$ thus identifying $V$ with $(1, V, 1)$ (the latter is a subalgebra of the semigroup algebra of the Brandt semigroup we work with). Then the vector space $\hat{V}=\oplus_{k=1}^{m_{i}}(k, V, 1)$ is a simple $S$-module, corresponding to $V$, and by construction is a direct summand of $V_{S}^{i}$. We have

$$
\operatorname{dim}(\hat{V})=m_{i} \cdot \operatorname{dim}(V)
$$

and hence $V_{S}^{i}$ is isomorphic to the multiplicity-free direct sum of all $\hat{V}$, where $V$ runs through the set of all simple $G_{e_{i}}$-modules. This completes the proof. 
Theorem 4 applies to many semigroups. Here are some examples:

- The symmetric inverse semigroup $\mathcal{I S}_{n}$ of all partial injections on $\{1,2, \ldots, n\}$ (also called the rook monoid), see [GM3, 2.5]. The conditions are satisfied because of [GM3, 2.6 and 5.1].

- The dual symmetric inverse semigroup $\mathcal{I}_{n}^{*}$ (or the monoid of block bijections) from [FL]. The conditions are satisfied because of [FL, Theorem $2.2]$.

- The maximal factorizable submonoid of $\mathcal{I}_{n}^{*}$ (or the monoid of uniform block bijections) from [FL]. The conditions are satisfied because of [FL, Section 3].

- The factor power $\mathcal{F P}^{+}\left(S_{n}\right)$ from [GM1, GM2]. Unlike the previous examples, this semigroup in not inverse, moreover, it is not even regular. However, all the required conditions are satisfied because of [GM2, Theorem 1] and [Ma].

\section{Combinatorial Gelfand model for the Hecke algebra}

For a permutation $\pi \in S_{n}$ define the support of $\pi$ as follows:

$$
\operatorname{supp}(\pi)=\{x \in\{1,2, \ldots, n\}: \pi(x) \neq x\} .
$$

For $1 \leq i<n$ let $s_{i}$ denote the transposition $(i, i+1)$.

For $q \in \mathbb{C}^{*}$ consider the Hecke algebra $\mathbf{H}_{n}(q)$, which is a $\mathbb{C}$-algebra with generators $\left\{T_{i}: 1 \leq i<n\right\}$ and defining relations

$$
\begin{aligned}
\left(T_{i}-q\right)\left(T_{i}+1\right) & =0, & & 1 \leq i<n ; \\
T_{i} T_{j} & =T_{j} T_{i}, & & 1 \leq i<j-1<n-1 ; \\
T_{i} T_{i+1} T_{i} & =T_{i+1} T_{i} T_{i+1}, & & 1 \leq i<n-1 .
\end{aligned}
$$

We have $\mathbf{H}_{n}(1) \cong \mathbb{C} S_{n}$ canonically. Let $V_{n, q}$ denote the formal linear span of $\left\{\mathrm{I}_{w}: w \in \mathcal{I}_{n}\right\}$. The following theorem is a slightly modified version of [APR, Theorem 1.2.2], which is more adjusted to our purposes.

Theorem 5. Let $1 \leq i<n$ and $w \in S_{n}$ be an involution. The assignment

$$
T_{i} \cdot \mathrm{I}_{w}= \begin{cases}q \mathrm{I}_{w}, & i, i+1 \notin \operatorname{supp}(w) ; \\ -\mathrm{I}_{w}, & i, i+1 \in \operatorname{supp}(w) ; \\ \mathrm{I}_{s_{i} w s_{i}}, & i \in \operatorname{supp}(w), i+1 \notin \operatorname{supp}(w) ; \\ q \mathrm{I}_{s_{i} w s_{i}}+(q-1) \mathrm{I}_{w}, & i \notin \operatorname{supp}(w), i+1 \in \operatorname{supp}(w)\end{cases}
$$


defines on $V_{n, q}$ the structure of an $\mathbf{H}_{n}(q)$-module. If we additionally assume that $q$ is not a root of unity, then $V_{n, q}$ is a Gelfand model for $\mathbf{H}_{n}(q)$.

\section{Combinatorial Gelfand model for the $q$ - rook monoid algebra}

For $q \in \mathbb{C}^{*}$ the $q$-rook monoid algebra $\mathbf{I}_{n}(q)$ is defined (see [Ha]) as a $\mathbb{C}$ algebra with generators $\left\{T_{i}: 1 \leq i<n\right\} \cup\left\{P_{i}: 1 \leq i \leq n\right\}$ and defining relations (3), (41), (5) and

$$
\begin{aligned}
T_{i} P_{j} & =P_{j} T_{i}=q P_{j}, & & 1 \leq i<j \leq n \\
T_{i} P_{j} & =P_{j} T_{i}, & & 1 \leq j<i \leq n-1 ; \\
P_{i}^{2} & =P_{i}, & & 1 \leq i \leq n \\
P_{i+1} & =P_{i} T_{i} P_{i}-(q-1) P_{i}, & & 1 \leq i \leq n-1 .
\end{aligned}
$$

This is not a semigroup algebra of some " $q$-rook monoid" but rather a oneparameter $(q-)$ deformation of the semigroup algebra of the rook monoid, in particular, $\mathbf{I}_{n}(1) \cong \mathbb{C} \mathcal{I} \mathcal{S}_{n}$ canonically. For generic $q$ there is a (non-canonical) isomorphism $\mathbf{I}_{n}(q) \cong \mathbb{C I S}_{n}($ see $[\mathrm{So1}, \mathrm{So}, \mathrm{Pa}])$.

To proceed, we need to fix some notation. Let $X=\{1,2, \ldots, n\} . \mathcal{I S}_{n}$ acts on $X$ in the standard way by partial permutations. For a partial transformation $\alpha$ we denote by $\operatorname{dom}(\alpha)$ the domain of $\alpha$. For a subset $A$ of $X$ denote by $e_{A}$ the identity transformation of $A$, and by $G(A)$ the $\mathcal{H}$-class of $e_{A}$, which consists of all $\pi \in \mathcal{I S}_{n}$ whose domains and images are equal to $A$.

Set $V=V_{q}^{\mathcal{I} \mathcal{S}_{n}}$ to be the vector space with the basis $\mathrm{I}_{w}$, where $w$ is an involution of $\mathcal{I} \mathcal{S}_{n}$.

Let $A \subset X$. For every $\pi \in G(A)$ define $\psi_{A}(\pi) \in S_{n}$ as the element whose action on $A$ coincides with that of $\pi$, and which acts identically on the set $X \backslash A$. The map $\psi_{A}$ gives rise to a monomorphism $\bar{\psi}_{A}$ from the linear span of $\left\{\mathrm{I}_{w}: w \in \mathcal{I}_{G(A)}\right\}$ to $V_{n, q}$ defined on the basis via $\bar{\psi}_{A}\left(\mathrm{I}_{w}\right)=\mathrm{I}_{\psi(w)}$. If $i, i+1 \in A$ and $w \in G(A)$ is an involution, we define

$$
T_{i} \circ \mathrm{I}_{w}=\bar{\psi}_{A}^{-1}\left(T_{i} \cdot \bar{\psi}_{A}\left(\mathrm{I}_{w}\right)\right)
$$

where the action $T_{i} \cdot \mathrm{I}_{\psi_{A}(w)}$ is given by ([6).

Now, for every generator $T_{i}, 1 \leq i \leq n-1$, and $P_{i}, 1 \leq i \leq n$, of $\mathbf{I}_{n}(q)$ we define a linear transformation of $V$ as follows:

$$
T_{i} \cdot \mathrm{I}_{w}= \begin{cases}T_{i} \circ \mathrm{I}_{w}, & i, i+1 \in \operatorname{dom}(w) ; \\ q \mathrm{I}_{w}, & i, i+1 \notin \operatorname{dom}(w) ; \\ \mathrm{I}_{s_{i} w s_{i}}, & i \in \operatorname{dom}(w), i+1 \notin \operatorname{dom}(w) \\ q \mathrm{I}_{s_{i} w s_{i}}+(q-1) \mathrm{I}_{w}, & i \notin \operatorname{dom}(w), i+1 \in \operatorname{dom}(w) ;\end{cases}
$$




$$
P_{i} \cdot \mathrm{I}_{w}= \begin{cases}\mathrm{I}_{w}, & \operatorname{dom}(w) \subset\{i+1, \ldots, n\} ; \\ 0, & \operatorname{dom}(w) \not \subset\{i+1, \ldots, n\} .\end{cases}
$$

Theorem 6. The assignments (11) and (12) define on $V$ the structure of an $\mathbf{I}_{n}(q)$-module. If we additionally assume that $q$ is not a root of unity, then $V$ is a Gelfand model for $\mathbf{I}_{n}(q)$.

To prove the theorem we need some preparation. The group $S_{n}$ acts on $\mathcal{I}_{\mathcal{I S}}$ by conjugation. This action gives rise to an action of $S_{n}$ on $V$ defined as follows: $\pi \mathrm{I}_{w} \pi^{-1}=\mathrm{I}_{\pi w \pi^{-1}}, w \in \mathcal{I}_{\mathcal{I} \mathcal{S}_{n}}$. We will need the following technical lemma:

Lemma 7. Let $w \in \mathcal{I}_{\mathcal{I S}_{n}}$.

(a) If $i, i+1 \in \operatorname{dom}(w)$, then $\pi\left(T_{i} \circ \mathrm{I}_{w}\right) \pi^{-1}=T_{i+1} \circ \mathrm{I}_{\pi w \pi^{-1}}$ for any $\pi \in S_{n}$ such that $\pi(k)=k+1$ for $k=i, i+1$.

(b) If $i, i+1 \in \operatorname{dom}(w)$ and $|j-i|>1$, then $s_{j}\left(T_{i} \circ \mathrm{I}_{w}\right) s_{j}=T_{i} \circ \mathrm{I}_{s_{j} w s_{j}}$.

Proof. Let $\psi=\psi_{\operatorname{dom}(w)}$ and $\tau=\psi_{\operatorname{dom}\left(\pi w \pi^{-1}\right)}$. Applying the definition of $\circ$ one reduces the first equality to

$$
\pi\left(\bar{\psi}^{-1}\left(T_{i} \cdot \bar{\psi}\left(\mathrm{I}_{w}\right)\right)\right) \pi^{-1}=\bar{\tau}^{-1}\left(T_{i+1} \cdot \bar{\tau}\left(\mathrm{I}_{\pi w \pi^{-1}}\right)\right) .
$$

Observe that for $k=i, i+1$ we have $k \in \operatorname{supp}(\psi(w))$ if and only if $k+1 \in$ $\operatorname{supp}\left(\tau\left(\pi w \pi^{-1}\right)\right)$. It follows that if $T_{i} \cdot\left(\bar{\psi}\left(\mathrm{I}_{w}\right)\right)$ is a linear combination of some $\mathrm{I}_{\psi(u)}$ 's then $T_{i+1} \cdot\left(\bar{\tau}\left(\mathrm{I}_{\pi w \pi^{-1}}\right)\right)$ is the same linear combination of the corresponding $\mathrm{I}_{\tau\left(\pi u \pi^{-1}\right)}$ 's. Hence we are left to check the equality

$$
\left.\pi\left(\bar{\psi}^{-1}\left(\mathrm{I}_{\psi(u)}\right)\right) \pi^{-1}=\bar{\tau}^{-1}\left(\mathrm{I}_{\tau\left(\pi u \pi^{-1}\right)}\right)\right) .
$$

The latter equality reduces to $\pi \mathrm{I}_{u} \pi^{-1}=\mathrm{I}_{\pi u \pi^{-1}}$, which follows from the definitions. This proves (国).

To prove (b) we set $\psi=\psi_{\operatorname{dom}(w)}$ and $\tau=\psi_{\operatorname{dom}\left(s_{j} w s_{j}\right)}$. The required equality reduces to

$$
s_{j}\left(\bar{\psi}^{-1}\left(T_{i} \cdot \bar{\psi}\left(\mathrm{I}_{w}\right)\right)\right) s_{j}=\bar{\tau}^{-1}\left(T_{i} \cdot \bar{\tau}\left(\mathrm{I}_{s_{j} w s_{j}}\right)\right) .
$$

Observe that for $k=i, i+1$ we have $k \in \operatorname{supp}(\psi(w))$ if and only if $k \in$ $\operatorname{supp}\left(\tau\left(s_{j} w s_{j}\right)\right)$. It follows that if $T_{i} \cdot\left(\bar{\psi}\left(\mathrm{I}_{w}\right)\right)$ is a linear combination of some $\mathrm{I}_{\psi(u)}$ 's then $T_{i} \cdot\left(\bar{\tau}\left(\mathrm{I}_{s_{j} w s_{j}}\right)\right)$ is the same linear combination of the corresponding $\mathrm{I}_{\tau\left(s_{j} u s_{j}\right)}$ 's. Hence we are left to check the equality

$$
\left.s_{j}\left(\bar{\psi}^{-1}\left(\mathrm{I}_{\psi(u)}\right)\right) s_{j}=\bar{\tau}^{-1}\left(\mathrm{I}_{\tau\left(s_{j} u s_{j}\right)}\right)\right) .
$$

The latter equality reduces to $s_{j} \mathrm{I}_{u} s_{j}=\mathrm{I}_{s_{j} u s_{j}}$, which follows from the definitions. This completes the proof. 
Proof of Theorem 6. First we show that $V$ is indeed an $\mathbf{I}_{n}(q)$-module. For this we have to check the defining relations.

Relation (3). Observe that with respect to the fixed basis of $V$ the matrix corresponding to the action of $T_{i}$ is a direct sum of blocks of three possible types: $(q),(-1),\left(\begin{array}{ll}0 & q \\ 1 & q-1\end{array}\right)$. Each of these blocks satisfies (3).

Relation (4). There are sixteen possible cases depending on whether each of the elements $i, i+1, j, j+1$ belongs to $\operatorname{dom}(w)$ or not. The cases where $i, i+1 \notin \operatorname{dom}(w)$ or $j, j+1 \notin \operatorname{dom}(w)$ are trivial since then the action of $T_{i}$ or $T_{j}$ respectively just multiplies the vectors $\mathrm{I}_{w}, \mathrm{I}_{s_{i} w s_{i}}$, or $\mathrm{I}_{s_{j} w s_{j}}$ respectively by $q$.

As $i$ and $j$ appear in (4) symmetrically, we are left to consider six cases.

Case 1. $i, i+1, j, j+1 \in \operatorname{dom}(w)$. We have

$$
\mathrm{I}_{w} \stackrel{T_{i}}{\mapsto} T_{i} \circ \mathrm{I}_{w} \stackrel{T_{j}}{\mapsto} T_{j} \circ\left(T_{i} \circ \mathrm{I}_{w}\right), \quad \mathrm{I}_{w} \stackrel{T_{j}}{\mapsto} T_{j} \circ \mathrm{I}_{w} \stackrel{T_{i}}{\mapsto} T_{i} \circ\left(T_{j} \circ \mathrm{I}_{w}\right),
$$

and the claim follows applying the relation (4) for $\mathbf{H}_{n}(q)$ (Theorem 5).

Case 2. $i, i+1, j \in \operatorname{dom}(w), j+1 \notin \operatorname{dom}(w)$. We have

$$
\mathrm{I}_{w} \stackrel{T_{i}}{\mapsto} T_{i} \circ \mathrm{I}_{w} \stackrel{T_{j}}{\mapsto} s_{j}\left(T_{i} \circ \mathrm{I}_{w}\right) s_{j}, \quad \mathrm{I}_{w} \stackrel{T_{j}}{\mapsto} \mathrm{I}_{s_{j} w s_{j}} \stackrel{T_{i}}{\mapsto} T_{i} \circ \mathrm{I}_{s_{j} w s_{j}},
$$

and the claim follows from Lemma $7(\mathrm{~b})$.

Case 3. $i, i+1, j+1 \in \operatorname{dom}(w), j \notin \operatorname{dom}(w)$. We have

$$
\begin{gathered}
\mathrm{I}_{w} \stackrel{T_{i}}{\mapsto} T_{i} \circ \mathrm{I}_{w} \stackrel{T_{j}}{\mapsto} q s_{j}\left(T_{i} \circ \mathrm{I}_{w}\right) s_{j}+(q-1) T_{i} \circ \mathrm{I}_{w}, \\
\mathrm{I}_{w} \stackrel{T_{j}}{\mapsto} q \mathrm{I}_{s_{j} w s_{j}}+(q-1) \mathrm{I}_{w} \stackrel{T_{i}}{\mapsto} q T_{i} \circ\left(\mathrm{I}_{s_{j} w s_{j}}\right)+(q-1) T_{i} \circ \mathrm{I}_{w},
\end{gathered}
$$

and the claim follows from Lemma $7(\mathrm{~b})$.

Case 4. $i, j \in \operatorname{dom}(w), i+1, j+1 \notin \operatorname{dom}(w)$. We have

$$
\mathrm{I}_{w} \stackrel{T_{i}}{\mapsto} \mathrm{I}_{s_{i} w s_{i}} \stackrel{T_{j}}{\mapsto} \mathrm{I}_{s_{j} s_{i} w s_{i} s_{j}}, \quad \mathrm{I}_{w} \stackrel{T_{j}}{\mapsto} \mathrm{I}_{s_{j} w s_{j}} \stackrel{T_{i}}{\mapsto} \mathrm{I}_{s_{i} s_{j} w s_{j} s_{i}},
$$

and the claim follows as $s_{j} s_{i}=s_{i} s_{j}$.

Case 5. $i, j+1 \in \operatorname{dom}(w), i+1, j \notin \operatorname{dom}(w)$. We have

$$
\begin{gathered}
\mathrm{I}_{w} \stackrel{T_{i}}{\mapsto} \mathrm{I}_{s_{i} w s_{i}} \stackrel{T_{j}}{\mapsto} q \mathrm{I}_{s_{j} s_{i} w s_{i} s_{j}}+(q-1) \mathrm{I}_{s_{i} w s_{i}}, \\
\mathrm{I}_{w} \stackrel{T_{j}}{\mapsto} q \mathrm{I}_{s_{j} w s_{j}}+(q-1) \mathrm{I}_{w} \stackrel{T_{i}}{\mapsto} q \mathrm{I}_{s_{i} s_{j} w s_{j} s_{i}}+(q-1) \mathrm{I}_{s_{i} w s_{i}},
\end{gathered}
$$

and the claim follows as $s_{j} s_{i}=s_{i} s_{j}$. 
Case 6. $i+1, j+1 \in \operatorname{dom}(w), i, j \notin \operatorname{dom}(w)$. We have $\mathrm{I}_{w} \stackrel{T_{i}}{\mapsto} q \mathrm{I}_{s_{i} w s_{i}}+(q-1) \mathrm{I}_{w} \stackrel{T_{j}}{\mapsto} q^{2} \mathrm{I}_{s_{j} s_{i} w s_{i} s_{j}}+q(q-1) \mathrm{I}_{s_{i} w s_{i}}+q(q-1) \mathrm{I}_{s_{j} w s_{j}}+(q-1)^{2} \mathrm{I}_{w}$, $\mathrm{I}_{w} \stackrel{T_{j}}{\mapsto} q \mathrm{I}_{s_{j} w s_{j}}+(q-1) \mathrm{I}_{w} \stackrel{T_{i}}{\mapsto} q^{2} \mathrm{I}_{s_{i} s_{j} w s_{j} s_{i}}+q(q-1) \mathrm{I}_{s_{j} w s_{j}}+q(q-1) \mathrm{I}_{s_{i} w s_{i}}+(q-1)^{2} \mathrm{I}_{w}$, and the claim follows as $s_{j} s_{i}=s_{i} s_{j}$.

Relation (5) . We consider eight possible cases depending on whether the elements $i, i+1, i+2$ belong to $\operatorname{dom}(w)$ or not.

Case 1. $i, i+1, i+2 \in \operatorname{dom}(w)$. This follows immedeately from Theorem 5 ,

Case 2. $i, i+1 \in \operatorname{dom}(w), i+2 \notin \operatorname{dom}(w)$. We have

$$
\begin{gathered}
\mathrm{I}_{w} \stackrel{T_{i}}{\mapsto} T_{i} \circ \mathrm{I}_{w} \stackrel{T_{i+1}}{\mapsto} s_{i+1}\left(T_{i} \circ \mathrm{I}_{w}\right) s_{i+1} \stackrel{T_{i}}{\mapsto} s_{i} s_{i+1}\left(T_{i} \circ \mathrm{I}_{w}\right) s_{i+1} s_{i}, \\
\mathrm{I}_{w} \stackrel{T_{i+1}}{\mapsto} \mathrm{I}_{s_{i+1} w s_{i+1}} \stackrel{T_{i}}{\mapsto} \mathrm{I}_{s_{i} s_{i+1} w s_{i+1} s_{i}} \stackrel{T_{i+1}}{\mapsto} T_{i+1} \circ \mathrm{I}_{s_{i} s_{i+1} w s_{i+1} s_{i}},
\end{gathered}
$$

and the claim follows applying Lemma 7(国) for $\pi=s_{i} s_{i+1}$.

Case 3. $i, i+2 \in \operatorname{dom}(w), i+1 \notin \operatorname{dom}(w)$. We have

$$
\begin{gathered}
\mathrm{I}_{w} \stackrel{T_{i}}{\mapsto} \mathrm{I}_{s_{i} w s_{i}} \stackrel{T_{i+1}}{\mapsto} T_{i+1} \circ \mathrm{I}_{s_{i} w s_{i}} \stackrel{T_{i}}{\mapsto} q s_{i}\left(T_{i+1} \circ \mathrm{I}_{s_{i} w s_{i}}\right) s_{i}+(q-1) T_{i+1} \circ \mathrm{I}_{s_{i} w s_{i}}, \\
\mathrm{I}_{w} \stackrel{T_{i+1}}{\mapsto} q \mathrm{I}_{s_{i+1} w s_{i+1}}+(q-1) \mathrm{I}_{w} \stackrel{T_{i}}{\mapsto} q T_{i} \circ \mathrm{I}_{s_{i+1} w s_{i+1}}+(q-1) \mathrm{I}_{s_{i} w s_{i}} \stackrel{T_{i+1}}{\mapsto} \\
q s_{i+1}\left(T_{i} \circ \mathrm{I}_{s_{i+1} w s_{i+1}}\right) s_{i+1}+(q-1) T_{i+1} \circ \mathrm{I}_{s_{i} w s_{i}} .
\end{gathered}
$$

The claim now follows from the equality

$$
s_{i} s_{i+1}\left(T_{i} \circ \mathrm{I}_{s_{i+1} w s_{i+1}}\right) s_{i+1} s_{i}=T_{i+1} \circ \mathrm{I}_{s_{i} w s_{i}},
$$

which, in turn, follows from Lemma 7((a)).

Case 4. $i+1, i+2 \in \operatorname{dom}(w), i \notin \operatorname{dom}(w)$. We have

$$
\begin{gathered}
\mathrm{I}_{w} \stackrel{T_{i}}{\mapsto} q \mathrm{I}_{s_{i} w s_{i}}+(q-1) \mathrm{I}_{w} \stackrel{T_{i+1}}{\mapsto} q^{2} \mathrm{I}_{s_{i+1} s_{i} w s_{i} s_{i+1}}+q(q-1) \mathrm{I}_{s_{i} w s_{i}}+(q-1) T_{i+1} \circ \mathrm{I}_{w} \\
\stackrel{T_{i}}{\mapsto} q^{2} T_{i} \circ \mathrm{I}_{s_{i+1} s_{i} w s_{i} s_{i+1}}+q(q-1) \mathrm{I}_{w}+(q-1) q s_{i}\left(T_{i+1} \circ \mathrm{I}_{w}\right) s_{i}+(q-1)^{2} T_{i+1} \circ \mathrm{I}_{w} \\
=: A+B+C+D, \quad(15) \\
\mathrm{I}_{w} \stackrel{T_{i+1}}{\mapsto} T_{i+1} \circ \mathrm{I}_{w} \stackrel{T_{i}}{\mapsto} q s_{i}\left(T_{i+1} \circ \mathrm{I}_{w}\right) s_{i}+(q-1) T_{i+1} \circ \mathrm{I}_{w} \stackrel{T_{i+1}}{\mapsto} \\
q^{2} s_{i+1} s_{i}\left(T_{i+1} \circ \mathrm{I}_{w}\right) s_{i} s_{i+1}+q(q-1) s_{i}\left(T_{i+1} \circ \mathrm{I}_{w}\right) s_{i}+ \\
+(q-1) T_{i+1} \circ\left(T_{i+1} \circ \mathrm{I}_{w}\right)=: E+F+G .
\end{gathered}
$$


We have $C=F$. Further, $B+D=G$ follows from (3). Finally, $A=E$ follows from Lemma 7(a) applying arguments similar to those used in the previous case.

Case 5. $i+2 \in \operatorname{dom}(w), i, i+1 \notin \operatorname{dom}(w)$. We have

$$
\begin{aligned}
& \mathrm{I}_{w} \stackrel{T_{i}}{\mapsto} q \mathrm{I}_{w} \stackrel{T_{i+1}}{\mapsto} q^{2} \mathrm{I}_{s_{i+1} w s_{i+1}}+q(q-1) \mathrm{I}_{w} \stackrel{T_{i}}{\longmapsto} \\
& q^{3} \mathrm{I}_{s_{i} s_{i+1} w s_{i+1} s_{i}}+q^{2}(q-1) \mathrm{I}_{s_{i+1} w s_{i+1}}+q^{2}(q-1) \mathrm{I}_{w}, \\
& \mathrm{I}_{w} \stackrel{T_{i+1}}{\mapsto} q \mathrm{I}_{s_{i+1} w s_{i+1}}+(q-1) \mathrm{I}_{w} \stackrel{T_{i}}{\mapsto} \\
& q^{2} \mathrm{I}_{s_{i} s_{i+1} w s_{i+1} s_{i}}+q(q-1) \mathrm{I}_{s_{i+1} w s_{i+1}}+q(q-1) \mathrm{I}_{w} \stackrel{T_{i+1}}{\longmapsto} \\
& q^{3} \mathrm{I}_{s_{i} s_{i+1} w s_{i+1} s_{i}}+q(q-1) \mathrm{I}_{w}+(q-1) q^{2} \mathrm{I}_{s_{i+1} w s_{i+1}}+(q-1)^{2} q \mathrm{I}_{w} \text {. }
\end{aligned}
$$

The right hand sides of (17) and (18) are equal.

Case 6. $i+1 \in \operatorname{dom}(w), i, i+2 \notin \operatorname{dom}(w)$. We have

$$
\begin{gathered}
\mathrm{I}_{w} \stackrel{T_{i}}{\mapsto} q \mathrm{I}_{s_{i} w s_{i}}+(q-1) \mathrm{I}_{w} \stackrel{T_{i+1}}{\mapsto} q^{2} \mathrm{I}_{s_{i} w s_{i}}+(q-1) \mathrm{I}_{s_{i+1} w s_{i+1}} \stackrel{T_{i}}{\mapsto} \\
q^{2} \mathrm{I}_{w}+q(q-1) \mathrm{I}_{s_{i+1} w s_{i+1}} ; \\
\mathrm{I}_{w} \stackrel{T_{i+1}}{\mapsto} \mathrm{I}_{s_{i+1} w s_{i+1}} \stackrel{T_{i}}{\mapsto} q \mathrm{I}_{s_{i+1} w s_{i+1}} \stackrel{T_{i+1}}{\mapsto} q^{2} \mathrm{I}_{w}+q(q-1) \mathrm{I}_{s_{i+1} w s_{i+1}} .
\end{gathered}
$$

Case \%. $i \in \operatorname{dom}(w), i+1, i+2 \notin \operatorname{dom}(w)$. In this case we have

$$
\begin{aligned}
& \mathrm{I}_{w} \stackrel{T_{i}}{\mapsto} \mathrm{I}_{s_{i} w s_{i}} \stackrel{T_{i+1}}{\mapsto} \mathrm{I}_{s_{i+1} s_{i} w s_{i} s_{i+1}} \stackrel{T_{i}}{\mapsto} q \mathrm{I}_{s_{i+1} s_{i} w s_{i} s_{i+1}} ; \\
& \mathrm{I}_{w} \stackrel{T_{i+1}}{\mapsto} q \mathrm{I}_{w} \stackrel{T_{i}}{\mapsto} q \mathrm{I}_{s_{i} w s_{i}} \stackrel{T_{i+1}}{\mapsto} q \mathrm{I}_{s_{i+1} s_{i} w s_{i} s_{i+1}} .
\end{aligned}
$$

Case 8. $i, i+1, i+2 \notin \operatorname{dom}(w)$. In this case the actions of both $T_{i} T_{i+1} T_{i}$ and $T_{i+1} T_{i} T_{i+1}$ map $\mathrm{I}_{w}$ to $q^{3} \mathrm{I}_{w}$.

Relation (7). We consider two possible cases.

Case 1. $\operatorname{dom}(w) \subset\{j+1, \ldots, n\}$. Then we also have $i, i+1 \notin \operatorname{dom}(w)$. Therefore, $T_{i} \cdot \mathrm{I}_{w}=q \mathrm{I}_{w}$ and thus $T_{i} P_{j} \cdot \mathrm{I}_{w}=P_{j} T_{i} \cdot \mathrm{I}_{w}=q P_{j} \cdot \mathrm{I}_{w}=q \mathrm{I}_{w}$.

Case 2. $\operatorname{dom}(w) \not \subset\{j+1, \ldots, n\}$. In this case it follows from the definitions that $T_{i} P_{j} \cdot \mathrm{I}_{w}=P_{j} T_{i} \cdot \mathrm{I}_{w}=q P_{j} \cdot \mathrm{I}_{w}=0$.

Relation (8). We consider two possible cases.

Case 1. $\operatorname{dom}(w) \subset\{j+1, \ldots, n\}$. Then $T_{i} P_{j} \cdot \mathrm{I}_{w}=P_{j} T_{i} \cdot \mathrm{I}_{w}=T_{i} \cdot \mathrm{I}_{w}$ as $T_{i} \cdot \mathrm{I}_{w}$ is a linear combination of $\mathrm{I}_{w}$ and $\mathrm{I}_{s_{i} w s_{i}}$ and both $\operatorname{dom}(w)$ and $\operatorname{dom}\left(s_{i} w s_{i}\right)$ are contained in $\{j+1, \ldots, n\}$.

Case 2. $\operatorname{dom}(w) \not \subset\{j+1, \ldots, n\}$. In this case $T_{i} P_{j} \cdot \mathrm{I}_{w}=P_{j} T_{i} \cdot \mathrm{I}_{w}=0$ follows from the definitions. 
Relation (9) follows immediately from the definitions.

Relation (10). Consider three possible cases.

Case 1. $\operatorname{dom}(w) \subset\{i+2, \ldots, n\}$. In this case we also have $i, i+1 \notin$ $\operatorname{dom}(w)$. Therefore, $T_{i} \cdot \mathrm{I}_{w}=q \mathrm{I}_{w}$ and hence

$$
P_{i+1} \cdot \mathrm{I}_{w}=\left(P_{i} T_{i} P_{i}-(q-1) P_{i}\right) \cdot \mathrm{I}_{w}=\mathrm{I}_{w} .
$$

Case 2. $\operatorname{dom}(w) \subset\{i+1, \ldots, n\}$ and $i+1 \in \operatorname{dom}(w)$. In this case $P_{i+1} \cdot \mathrm{I}_{w}=0$ and $P_{i} \cdot \mathrm{I}_{w}=\mathrm{I}_{w}$. We are left to check that $P_{i} T_{i} \cdot \mathrm{I}_{w}=(q-1) I_{w}$. Observe that $P_{i} \cdot \mathrm{I}_{s_{i} w s_{i}}=0$ as $\operatorname{dom}\left(s_{i} w s_{i}\right) \not \subset\{i+1, \ldots, n\}$. Taking this into account we obtain

$$
P_{i} T_{i} \cdot \mathrm{I}_{w}=P_{i} \cdot\left(q \mathrm{I}_{s_{i} w s_{i}}+(q-1) \mathrm{I}_{w}\right)=(q-1) \mathrm{I}_{w} .
$$

Case 3. $\operatorname{dom}(w) \not \subset\{i+1, \ldots, n\}$. This case follows directly from the definitions. This completes the proof of the fact that $V$ is an $\mathbf{I}_{n}(q)$-module.

To prove that $V$ is a Gelfand model for $\mathbf{I}_{n}(q)$ we use the results of [Pa] and arguments analogous to those used in the second part of the proof of Theorem 4. Set $J_{0}=\mathbf{I}_{n}(q), J_{k}=\mathbf{I}_{n}(q) P_{k} \mathbf{I}_{n}(q), k=1, \ldots, n$, and $J_{n+1}=0$. In $[\mathrm{Pa}$, Section 4] it is shown that there is an algebra isomorphism

$$
\mathbf{I}_{n}(q) \cong \bigoplus_{k=0}^{n} J_{k} / J_{k+1}
$$

and that $J_{k} / J_{k+1}$ is isomorphic to $\mathbf{M}_{l_{k}}(\mathbb{C}) \otimes \mathbf{H}_{n-k}(q)$ (where $\mathbf{M}_{l_{k}}(\mathbb{C})$ is the matrix algebra and $l_{k}=\left(\begin{array}{l}n \\ k\end{array}\right)$ ), with a multiplication, which is appropiately twisted to take into account powers of $q$, which may appear when multiplying in $J_{k} / J_{k+1}$. This twist can be forgotten using [Pa, Corollary 15].

For $k=0, \ldots, n$ let $V^{(k)}$ denote the subspace of $V$, spanned by all $\mathrm{I}_{w}$, where $|\operatorname{dom}(w)|=n-k$. Denote also by $\tilde{V}^{(k)}$ the subspace of $V^{(k)}$, spanned by all $\mathrm{I}_{w}$, where $\operatorname{dom}(w)=\{1,2, \ldots, n-k\}$. Finally, we identify $\mathbf{H}_{n-k}(q)$ with the subalgebra of $\mathbf{I}_{n}(q)$, generated by $T_{i}, i<n-k$. From the definitions we immediately obtain:

- $V=\oplus_{k=0}^{n} V^{(k)}$ as $\mathbf{I}_{n}(q)$-modules;

- $J_{k+1} \cdot V^{(k)}=0$.

Furthermore, from the definitions we have that $\tilde{V}^{(k)}$ is an $\mathbf{H}_{n-k}(q)$-module and, moreover, from Theorem 5 we have that $\tilde{V}^{(k)}$ is a Gelfand model of $\mathbf{H}_{n-k}(q)$.

As $\operatorname{dim} V^{(k)}=l_{k} \cdot \operatorname{dim} \tilde{V}^{(k)}$, we obtain that $V^{(k)}$ is a Gelfand model for $\mathrm{M}_{l_{k}}(\mathbb{C}) \otimes \mathbf{H}_{n-k}(q)$. Adding everything up we thus get that $V$ is a Gelfand model for $\mathbf{I}_{n}(q)$. This completes the proof. 


\section{References}

[APR] R. Adin, A. Postnikov, Y. Roichman, Combinatorial Gelfand model, preprint math.RT arXiv:0709.3962, to appear in J. Algebra.

[CP] A. Clifford, G. Preston, The algebraic theory of semigroups, vol I. Amer. Math. Soc. Surveys 7 (Providence, R.I.) 1961.

[DHP] M. Dieng, T. Halverson, V. Poladian, Character formulas for $q$-rook monoid algebras, J.Algebraic Combin. 17 (2003), 99-123.

[IRS] N. Inglis, R. Richardson, J. Saxl, An explicit model for the complex representations of $S_{n}$. Arch. Math. (Basel) 54 (1990), no. 3, 258-259.

[FL] D. FitzGerald, J. Leech, Dual symmetric inverse monoids and representation theory. J. Austral. Math. Soc. Ser. A 64 (1998), no. 3, $345-367$.

[GM1] A. Ganyushkin, V. Mazorchuk, Factor powers of semigroups of transformations. Dopov./Dokl. Akad. Nauk Ukraïni 1993, no. 12, 5-9 (1994).

[GM2] A. Ganyushkin, V. Mazorchuk, The structure of subsemigroups of factor powers of finite symmetric groups. Math. Notes 58 (1995), no. 3-4, 910-920 (1996).

[GM3] O. Ganyushkin, V. Mazorchuk, Classical finite transformation semigroups, An introduction. Algebra and Applications, Vol. 9, Springer Verlag, to appear in 2008.

[GMS] O. Ganyushkin, V. Mazorchuk, B. Steinberg, On the irreducible representations of a finite semigroup, preprint math.RT arXiv:0712.2076.

[Ha] T. Halverson, Representations of the q-rook monoid. J. Algebra 273 (2004), no. 1, 227-251.

[HR] T. Halverson, A. Ram, q-rook monoid algebras, Hecke algebras, and Schur-Weyl duality. J. Math. Sci. (N. Y.) 121 (2004), no. 3, 24192436

[Hi] P. Higgins, Techniques of semigroup theory. Oxford Science Publications. The Clarendon Press, Oxford University Press, New York, 1992. 
[Ma] V. Mazorchuk, Green's relations on $\mathcal{F P}^{+}\left(S_{n}\right)$. Mat. Stud. 15 (2001), no. 2, 151-155.

[Pa] R. Paget, Representation theory of $q$-rook monoid algebras. J. Algebraic Combin. 24 (2006), no. 3, 239-252.

[Sa] B. Sagan, The symmetric group. Representations, combinatorial algorithms, and symmetric functions. Second edition. Graduate Texts in Mathematics, 203. Springer-Verlag, New York, 2001.

[So1] L. Solomon, The Bruhat decomposition, Tits system and Iwahori ring for the monoid of matrices over a finite field, Geom. Dedicata 36 (1990), 15-49.

[So] L. Solomon, The Iwahori algebra of $\mathbf{M}_{n}\left(\mathbf{F}_{q}\right)$. A presentation and a representation on tensor space. J. Algebra 273 (2004), no. 1, 206226.

G.K.: Department of Mechanics and Mathematics, Kyiv Taras Shevchenko University, 64, Volodymyrska st., 01033, Kyiv, UKRAINE, e-mail: akudr@univ.kiev.ua

V.M.: Department of Mathematics, Uppsala University, SE 471 06, Uppsala, SWEDEN, e-mail: mazor@math.uu.se 